\title{
Preliminary Studies of Dynamics of Physical Agents Ecosystems
}

\author{
Josep Lluís de la Rosa, Israel Muñoz, Bianca Innocenti, Albert Figueras, Miquel \\ Montaner, Josep Antoni Ramon \\ Institut d'Informàtica i Aplicacions, Universitat de Girona \& LEA-SICA \\ Lluis Santaló s/n, E-17071 Girona, Catalonia \\ \{peplluis, imunoz, bianca, figueras, montaner, jar\}@ eia.udg.es
}

\begin{abstract}
This paper is a step forward from the agent ecosystems that Hogg studied [6]. We plan to extend these agents ecosystems to physical agents that interact with the physical world. The aim is to conceive algorithms for the choice of resources and to expand this work. Dynamics of choice in such ecosystems depends on pay-off functions that contain information about the real physical world. One contribution here is to formalise the choice of knowledge resources by including a consensus technique. The second contribution is to include diversity by means of physical agents and to analyse the emergent impact in terms of diversity and performance. Simulated soccer robots exemplifies all this.
\end{abstract}

\section{Introduction}

\subsection{Consensus}

There is extensive research in consensus and sensor fusion techniques, in which normally consensus is more focused on knowledge and sensor fusion in the acquisition of data, [1] and [3]. The idea is simple, when there are multiple sources of information or knowledge, there will possibly be contradictions that will require additional knowledge and techniques to obtain useful knowledge out of the contradictory data.

\subsection{Physical Agents}

According to Asada [2], physical bodies play an important role, when emerging complex behaviours in order to achieve goals in dynamic real world. The RoboCup Physical Agent Challenge provides a good testbed to see how physical bodies play a significant role in realising intelligent behaviour using RoboCup framework [7]. Our research focuses in dynamical physical agents, in which the term dynamics is a way to model the transient and steady behaviours of the physical bodies of agents [8]. 


\subsection{Universal Information Ecosystems}

Hogg and Hubermann [6] studied the dynamics of choice in communities of agents, with different delays to access information. This work defined the resource choice as a preference function of resource 1 vs. resource 2 . It is defined in terms of difference of the performance obtained by applying the information from resource 1 and 2 . The uncertainty $(\sigma)$ is modelled as a typical deviation on performance to decide when clearly resource $1 / 2$ leads the agent to a better performance.

$$
\begin{gathered}
\rho=\frac{1}{2} \cdot\left(1+e r f\left(\frac{G_{1}\left(F\left(f_{1}\right)\right)-G_{2}\left(F\left(f_{2}\right)\right)}{2 \sigma}\right)\right) \\
\frac{d f_{r s}}{d t}=\alpha\left(f_{s}^{t y p e} \rho_{r s}-f_{r s}\right)
\end{gathered}
$$

$F\left(f_{i}\right)$ is a reward function related to the resource usage. $G$ has access to $F\left(f_{i}\right)$ with different delays. $\alpha$ is the rate at which agents re-evaluate their resource choice. $f_{r s}$ is the rate of agents of type $s$ that at $t$ prefer resource $r$, also called population for $r, s$

\subsection{Work to be Developed}

All these concepts have been applied to a team of agents, implemented in simulation using JavaSoccer. A set of agents, with different physical features, will play against a benchmark. Each player at any time takes a decision based on the rules, coded in 5 rule sets or roles, that goes through a consensus process, to reduce conflicts. Consensus parameters are related to the evolution of the different populations shown in [6].

\section{Description of the Benchmark}

Implicit opponent is a generalisation of benchmarks that were proposed by Jeffrey Johnson [5]. Its features allow having a good reference benchmark so that all existing teams can be evaluated/compared in a robust and general way. This benchmark has two main features. The first feature is an angled field, this causes the ball to fall down, in case it is not properly controlled, this is a challenging difficulty. The second feature is the presence of static obstacles. The robot can not easily move the ball to the opponent goal and it hinders the player to regain the ball [9].

\section{Realisation of Decision Making}

Five roles have been coded using fuzzy sets. These roles propose actions with a certainty associated, considering the position of the ball and the position of the player, 
which is making the decision. These roles are: goalie $(G)$, defender (D), right/left mid-fielders (RM/LM) and forward (F). Each role has a number of actions associated, and some roles share one or more actions. Actions have to go through a consensus process, in order to reduce conflicts, in case two agents decide to do the same. The procedure is the following: initially each robot takes the decision with the highest certainty associated after the revision process done by the consensus technique. In case two agents want to take the same decision, the one with the highest certainty wins. The revision process is based on two parameters, Prestige and Necessity [4], that modify the certainty $(\varphi)$.

Prestige $(P)$ performs a linear transformation over $\varphi$

$$
\varphi^{\prime}=P(\varphi)=P \cdot \varphi
$$

Then, Necessity $(N)$ performs a non-linear transformation over $\varphi^{\prime}$

$$
\varphi^{\prime \prime}=N(P(\varphi))
$$

\section{A Consensus Based Choice Function in Ecosystems}

In this paper Hogg's work is used to increase or diminish the preference of every agent for each of the resources. In this case resources $(r)$ are roles and agent diversity $(s)$ is created through different physical features for each robot, instead of using delays to evaluate pay-off. Equation (1) has been modified in order to deal with 5 resources. This equation is a first approximation and it will be improved in the future.

$$
\rho_{r s}=\frac{G_{r s}}{\sum_{r=1}^{R} G_{r s}}
$$

The agent $s$ has a performance $G r$ for each resource $r$, which is calculated in a temporal window of $T_{w}$ units of time. This index is calculated taking into account several aspects: set points accomplishment, player situation on the field depending on the role, goals received/scores, dribbling ability, ability to regain the ball. Each rewarding action has a value associated depending on the position on the field, where the action has taken place. For example, it is far more important to regain the ball at the penalty area than recovering it at midfield. $f_{s}$ changes according to this equation:

$$
\begin{gathered}
\frac{d f_{s}^{\text {type }}}{d t}=\gamma\left(\eta_{s}-f_{s}^{\text {type }}\right) \\
\eta_{s}=\frac{\sum_{r=1}^{R} G_{r s}}{\sum_{s=1}^{S} \sum_{r=1}^{R} G_{r s}}
\end{gathered}
$$


These equations were also proposed by Hogg [5], but also they have been modified. $\eta_{s}$ increases population values to agents that perform better globally.

In order to combine the idea of preference for one role and the population values, the value of $f r s$ is used to assign the parameter Prestige $(P)$ for revision in consensus. ( $k$ is a constant value).

$$
P_{r s}=\frac{1}{1+\exp \left(\frac{1}{\frac{N_{-} \text {players } \cdot N_{\text {_roles }}}{k}-f_{r s}}\right)}
$$

The values of Necessity are computed depending on the resources/roles use. It aims at having a team with balanced resource usage, this introduces some kind of continuos excitement in the system. The way the value of Necessity is computed as follows: ( $p r$ is the fraction of resource $r$ usage and $\delta$ is a constant value)

$$
N_{r}=\exp \left(-\delta \cdot \frac{p_{r}}{f_{r s}}\right)
$$

\section{Experiments}

Using the ideas presented so far, the experiments performed aim at showing how a system, made up of different types of physical agents, can self-organise/stabilise (stable means show persistence on one role) depending on their fitness of their physical features for each role. Also the experiments try to show how a diversity of physical agents help to stabilise the system vs. a team of identical agents under some circumstances

\subsection{Experimental Conditions}

The experiments were performed under the same conditions:

\begin{tabular}{ccccccc}
\hline Time & $\mathrm{Tw}$ & $\Delta \mathrm{t}$ & $\mathrm{k}$ & $\gamma$ & $\delta$ & Field angle \\
\hline $12 \mathrm{~h}$ & $100 \mathrm{sec}$ & $0.01-0.05$ & 0.02 & 1 & 1 & $8^{\mathrm{o}}$ \\
\hline
\end{tabular}

Table 1. Parameter Values

Time is JavaSoccer simulation time (sample time $100 \mathrm{~ms}$ ). The differential equations 4 and 7 have been approximated to a discrete formulation .Initially $\Delta t$ is a very small (0.01), after some time this value increases progressively until 0.05 . This change allows the agents to go through many different situations initially, until they "decide" to settle in one role. The used agents have the following features: 


\begin{tabular}{ccccc}
\hline Agent & Radius (cm) & $\begin{array}{c}\text { Translation } \\
\text { Velocity }(\mathrm{m} / \mathrm{s})\end{array}$ & $\begin{array}{c}\text { Rotational } \\
\text { Velocity (rad/s) }\end{array}$ & $\begin{array}{c}\text { Kick } \\
\text { Speed (m/s) }\end{array}$ \\
\hline 0 & 0.08 & 0.15 & 3.28 & 0.85 \\
1 & 0.04 & 0.40 & 8.28 & 0.2 \\
2 & 0.07 & 0.20 & 4.28 & 0.65 \\
3 & 0.06 & 0.30 & 6.28 & 0.5 \\
4 & 0.05 & 0.35 & 7.28 & 0.3 \\
\hline
\end{tabular}

Table 2. Robot Features

\subsection{Experimental Results}

\begin{tabular}{cccccc}
\hline Agent & Final Role & $\begin{array}{c}\text { Dominant } \\
\text { Population }\end{array}$ & $\begin{array}{c}\text { \% Final Role } \\
\text { Usage }\end{array}$ & $\begin{array}{c}\text { \% Dominant } \\
\text { Population }\end{array}$ & $\begin{array}{c}\text { Stable } \\
\text { Roles ? }\end{array}$ \\
\hline 0 & D/G & D/G & $50 / 100$ & $75 / 98$ & Y/Y \\
1 & F/No Role & F/LM & $95 /--$ & $58 / 60$ & Y/N \\
2 & G/LM & G/LM & $100 / 90$ & $98 / 63$ & Y/Y \\
3 & LM/RM & LM/RM & $95 / 90$ & $66 / 63$ & Y/Y \\
4 & RM/No Role & RM/D & $90 /--$ & $48 / 36$ & Y/N \\
\hline
\end{tabular}

Table 3. Results for heterogeneous/homogeneous agents

These results are specific for one run, although these results can be obtained in many runs. As it can be seen from Table 3 heterogeneous agents tend to self-organise taking over each one a role. There is a very interesting aspect to notice: in logged data agents 1 and 4 seem to take a role, but after some time they finally settle in a different role. The reason for this sudden change is the better fitness of agent 1 for forward role. Agent 1 presents an excellent dribbling ability. Agents 3 and 4 have a quite good dribbling ability but they tend to regain very well balls at mid-field. For defensive roles it can be seen that agent 2 is the best for goalie, this is because agent 2 is big but at the same time is faster than 1 . Agent 0 has a relatively small role usage, the reason for that problem is the small number of actions available for all roles. This problem leads to many conflicts among agents and it is manifested in defensive roles. For the experiments performed over the identical agents (agent type 3), results show that some agents tend to take a role, but there are other agents that do not seem to find a role, taking continuously rules from different roles. The reason for this problem seems to be the difficulty to find an agent fitted for defensive and forward roles, under the extreme circumstances that the experiments have been performed. In this case seems that diversity helps to stabilise the system, although this is not always true. Some experiments performed with a smaller angle show that both multi-agent systems tend to self-organised, taking each agent a different role. 


\section{Conclusions}

We have been able to expand Hogg's work [6] to physical agents, although some changes were needed to adapt these ideas to physical agents. Also we have been able to link the dynamics of choice and the evolution of populations to a set of parameters that features the consensus technique here presented in a successfully way and finally we have introduced a new type of diversity based on physical features.

\section{Future Work}

All these ideas will be implemented in the future using real robots and the benchmark proposed. These robots, although physically identical, will have a different dynamical behaviour, achieved using different types of control. Reward assignment, equations, and so on and forth will be improved in the future.

\section{Acknowledgement}

This work is partially funded by projects TAP98-0955-C03-02, TAP99-1354-E, of the Spanish Research Foundation CICYT, and the special action 2000ACES00018.

\section{References}

1. Abidi M.A and R.C. González, Data Fusion in Robotics \&Machine Intelligence, Academic Press, pp:7-135, 1992.

2. Asada M., Kuniyoshi Y., et al. The RoboCup Physical Agent Challenge, First RoboCup Workshop in the XV IJCAI-97 International Joint Conference on Artificial Intelligence, pp.51-56, 1997.

3. K. Chi Ng and B. Abramson, Consensus Diagnosis: A Simulation Study, IEEE Transactions on Systems, Man, and Cybernetics, Vol. 22, n 5, September/October 1992.

4. de la Rosa J. Ll. and Delgado A. Fuzzy Evaluation of Information Received from other Systems, Proceedings of the IPMU'92, pp. 769-778, Palma de Mallorca, July 6-10, 1992.

5. Johnson J., de la Rosa J.Ll. and Kim J.H., "Benchmark Tests in the Science of Robot Football" Proceedings IEEE of Mirosot-98, Paris 1998.

6. Hogg T. and Huberman B. A., Controlling Chaos in Distributed Systems, IEEE Transactions on Systems, Man, and Cybernetics, Vol. 21, No. 6, November/December 1991.

7. Kitano H., Veloso M., et al., The RoboCup Synthetic Agent Challenge 97, XV IJCAI-97 Int. Joint Conference on Artificial Intelligence, Vol 1, pp.24-29, Nagoya, August 1997.

8. Oller A, de la Rosa J.Ll., and del Acebo E., DPA2: Architecture for Co-operative Dynamical Physical Agents, MAMAAW '99, Valencia June 1999.

9. de la Rosa J. Ll., Muñoz I., Duhaut D., Johnson J., Experimental Basis for Benchmarking RoboCup, Proceedings of the first spanish-portugese Workshop on Physical Agents, pp87101, Tarragona, September 2000. 